\title{
Remarks on the blow-up for the Schrödinger equation with critical mass on a plane domain
}

\author{
Valeria Banica
}

\begin{abstract}
We concentrate on the analysis of the critical mass blowing-up solutions for the cubic focusing Schrödinger equation with Dirichlet boundary conditions, posed on a plane domain. We bound from below the blow-up rate for bounded and unbounded domains. If the blow-up occurs on the boundary, the blow-up rate is proved to grow faster than $(T-t)^{-1}$, the expected one. Moreover, we state that blow-up cannot occur on the boundary, under certain geometric conditions on the domain.
\end{abstract}

\section{Introduction}

In this paper we study the blow-up of critical mass solution to cubic focusing NLS in a plane domain with Dirichel boundary condition. First we recall the classical results on the whole euclidean plane. These results can be generalized in higher dimension.

Consider the nonlinear Schrödinger equation on $\mathbb{R}^{2}$

$$
(S)\left\{\begin{array}{c}
i \partial_{t} u+\Delta u+|u|^{p-1} u=0 \\
u(0)=u_{0}
\end{array}\right.
$$

The associated Cauchy problem is locally well posed in $\mathbb{H}^{1}$ for all $p$ ([6], [8]).

The Gagliardo-Nirenberg inequality

$$
\|v\|_{p+1}^{p+1} \leq C_{p+1}\|v\|_{2}^{2}\|\nabla v\|_{2}^{p-1}
$$

implies that the energy of the solution $u$ of the equation $(S)$,

$$
E(u)=\frac{1}{2} \int_{\mathbb{R}^{2}}|\nabla u|^{2} d x-\frac{1}{p+1} \int_{\mathbb{R}^{2}}|u|^{p+1} d x
$$

MSC 2000: 35Q55, 35B33, 35B40, 35Q40.

Keywords : nonlinear Schrödinger, blow-up. 
is bounded from below by

$$
\|\nabla u\|_{2}^{2}\left(\frac{1}{2}-\frac{C_{p+1}}{p+1}\|u\|_{2}^{2}\|\nabla u\|_{2}^{p-3}\right) .
$$

As a consequence, if $p<3$, since the mass is conserved, the gradient of $u$ is controlled by the energy. Therefore the solution does not blow up and global existence occurs.

The cubic power is a critical power, in the sense that the nonlinearity is strong enough to generate solutions blowing up in a finite time. However, even in this case, we have a global result for small initial conditions.

Indeed, in the case, if the mass of the initial condition is small enough so that

$$
\frac{C_{4}}{4}\|u\|_{2}^{2}<\frac{1}{2}
$$

then the energy controls the gradient and again, the global existence is proved for the equation $(S)$.

For this particular value of $p$, Weinstein has given a sharpening of the GagliardoNirenberg inequality ([20]). By variational methods using Lions concentrationcompacity lemma ([11], [12]), he obtained the existence of a minimizer $Q$ for the optimal constant of Gagliardo-Nirenberg's inequality

$$
\frac{1}{C_{4}}=\inf _{v \in \mathbb{H}^{1}\left(\mathbb{R}^{2}\right)} \frac{\|v\|_{2}^{2}\|\nabla v\|_{2}^{2}}{\|v\|_{4}^{4}}
$$

This minimizer verifies the Euler-Lagrange equation

$$
\Delta Q+Q^{3}=Q
$$

Such a positive function, called ground state of the nonlinear Schrödinger equation, is radial, exponentially decreasing at infinity and regular. Recently, Kwong has shown that it is unique up to a translation ([10]). Moreover, it verifies the Pohozaev identities

$$
\left\{\begin{array}{c}
\|\nabla Q\|_{2}^{2}-\|Q\|_{4}^{4}+\|Q\|_{2}^{2}=0 \\
\|Q\|_{4}^{4}=2\|Q\|_{2}^{2}
\end{array},\right.
$$

leading to the relations between the norms of $Q$ which give the optimal value for the constant of the Gagliardo-Nirenberg inequality

$$
C_{4}=2 \frac{1}{\|Q\|_{2}^{2}}
$$

In conclusion, if $p=3$, the solutions of the equation $(S)$ with initial condition of mass smaller than the one of the ground state

$$
\|u\|_{2}<\|Q\|_{2}
$$

are global in time.

The mass $\|Q\|_{2}$ is critical, in the sense that we can construct as follows solutions of mass equal to $\|Q\|_{2}$, which blows up in finite time. Since $p=3$, the pseudoconformal transform of a solution $u$ of $(S)$

$$
\frac{1}{t} e^{i \frac{|x|^{2}}{4 t}} u\left(-\frac{1}{t}, \frac{x}{t}\right)
$$


is also a solution of $(S)([4])$. So, from a stationary solution on $\mathbb{R}^{2}$

$$
e^{i t} Q(x)
$$

for all positive $T$,

$$
u(t, x)=\frac{e^{\frac{i}{T-t}}}{T-t} e^{-i \frac{|x|^{2}}{4(T-t)}} Q\left(\frac{x}{T-t}\right),
$$

is a solution blowing-up at the time $T$. Moreover, Merle proved in [14] that all blowing up solutions on $\mathbb{R}^{2}$ with critical mass $\|Q\|_{2}$ are of this type, up to the invariants of the equation. The proof is based on a result of concentration of Weinstein ([21], see Lemma 1.2) and on the study of the first order momentum

$$
f(t)=\int_{\mathbb{R}^{2}}|u(t, x)|^{2} x d x
$$

and of the virial

$$
g(t)=\int_{\mathbb{R}^{2}}|u(t, x)|^{2}|x|^{2} d x
$$

associated to a solution $u$ of the equation $(S)$. The conservative properties of these two quantities on $\mathbb{R}^{2}$, in the case of the critical power 3 , play an important role in Merle's proof. The derivative of the first order momentum is constant in time

$$
\partial_{t}^{2} f=0,
$$

and $g$ satisfies the virial identity $([4])$

$$
\partial_{t}^{2} g=16 E(u) .
$$

In certain cases of initial conditions with mass larger than $\|Q\|_{2}$ recent achievements were done by Merle and Raphaël ([15], [16]).

In this work we are concerned with the nonlinear Schrödinger equation posed on a regular domain $\Omega$ of $\mathbb{R}^{2}$, with Dirichlet boundary conditions

$$
\left\{\begin{array}{c}
i \partial_{t} u+\Delta u+|u|^{p-1} u=0 \\
u_{\mid \mathbb{R} \times \partial \Omega}=0 \\
u(0)=u_{0}
\end{array} .\right.
$$

The Cauchy problem is well posed on $\mathbb{H}^{2} \cap \mathbb{H}_{0}^{1}(\Omega)$. For nonlinearities less than cubic, Vladimirov [19] and Ogawa and Ozawa [17] have shown the well-posedness of the Cauchy problem on $\mathbb{H}_{0}^{1}(\Omega)$, but without the uniform continuity of the flow on bounded sets of $\mathbb{H}_{0}^{1}(\Omega)$. For nonlinearities stronger than cubic in dimension 2, or for any power nonlinearity $p$, in dimension higher than 2, the Cauchy problem on $\mathbb{H}_{0}^{1}(\Omega)$ is open.

For the equation with power $p<3$, one can show as for the case $\mathbb{R}^{n}$ that the $\mathbb{H}_{0}^{1}(\Omega)$ solutions are global in time. For the equation with power $p \geq 3$, posed on a star-shaped domain of $\mathbb{R}^{n}$, Kavian has proved the blow-up in finite time of the $\mathbb{H}^{2} \cap \mathbb{H}_{0}^{1}(\Omega)$ solutions of negative energy or of positive energy but under some conditions on the first and second derivatives of the virial ([9]). His proof follows 
the ones on $\mathbb{R}^{n}$ of Zakharov ([23]) and Glassey ([7]), by estimating via the geometric condition on $\Omega$ the boundary terms which appear in the second derivative of the virial. The proof is based on an upper bound of the virial in terms of its first and second derivative, which implies the cancellation of the virial at a finite time $T$. Since the mass is conserved, it follows that the solution must blow up at the time $T$.

From now on we shall analyze the cubic equation on $\Omega$

$$
\left(S_{\Omega}\right) \quad\left\{\begin{array}{c}
i \partial_{t} u+\Delta u+|u|^{2} u=0 \\
u_{\mid \mathbb{R} \times \partial \Omega}=0 \\
u(0)=u_{0}
\end{array} .\right.
$$

The usual Strichartz inequalities are no longer valid and the loss of derivatives is stronger than in the case of a compact manifold ([3]).

As in the case of the plane, for initial conditions with mass smaller than the one of the ground state, the Cauchy problem is globally well-posed on $\mathbb{H}^{2} \cap \mathbb{H}_{0}^{1}(\Omega)$. The proof, given by Brézis and Gallouët, is based on logarithmic type estimates ([2]). This result has been extended to the natural space $\mathbb{H}_{0}^{1}(\Omega)$, up to the uniform continuity of the flow ([19],[17],[4]).

The critical mass for blow-up is $\|Q\|_{2}$, as in the case of the equation posed on $\mathbb{R}^{2}$. More precisely, the following result holds.

Theorem 1.1 (Burq-Gérard-Tzvetkov [3]) Let $\Omega$ be a regular bounded domain of $\mathbb{R}^{2}$. Let $x_{0} \in \Omega$ and $\psi \in \mathcal{C}_{0}^{\infty}$ be a function equal to 1 near $x_{0}$. Then there exist positive numbers $\kappa$ and $\alpha_{0}$ such that for all $\alpha>\alpha_{0}$, there exists a time $T_{\alpha}$ and $a$ function $r_{\alpha}$ defined on $\left[0, T_{\lambda}[\times \Omega\right.$, satisfying

$$
\left\|r_{\alpha}(t)\right\|_{\mathbb{H}^{2}(\Omega)} \leq c e^{-\frac{\kappa}{T_{\alpha}-t}}
$$

such that

$$
u(t, x)=\psi(x) \frac{e^{\frac{i}{\alpha^{2}\left(T_{\alpha}-t\right)}}}{\alpha\left(T_{\alpha}-t\right)} e^{-i \frac{\left|x-x_{0}\right|^{2}}{4 \alpha\left(T_{\alpha}-t\right)}} Q\left(\frac{x-x_{0}}{\alpha\left(T_{\alpha}-t\right)}\right)+r_{\alpha}(t, x),
$$

is a critical mass solution of $\left(S_{\Omega}\right)$, blowing up at $x_{0}$ at the time $T_{\alpha}$ with the blow-up rate $\frac{1}{T_{\alpha}-t}$.

The proof, following an idea of Ogawa and Tsutsumi ([18]), is based on a fixed point method wich allows to complete the cut-off of the explicit blowing up solution on $\mathbb{R}^{2}$ at $x_{0}$ to a blowing up solution on $\Omega$ at $x_{0}$. Theorem 4.1.1 implies in particular that at every point of $\Omega$ there are explosive solutions. Moreover, the proof is still valid for the torus $\mathbb{T}^{2}$ and for a larger class of subsets of the plane, which satisfy the property of 2-continuation, from $\mathbb{H}^{2} \cap \mathbb{H}_{0}^{1}(\Omega)$ to $\mathbb{H}^{2}\left(\mathbb{R}^{2}\right)$, and for which the Laplacian domain

$$
D\left(-\Delta_{\Omega}\right)=\left\{u \in \mathbb{H}_{0}^{1}(\Omega), \Delta u \in \mathbb{L}^{2}(\Omega)\right\},
$$

is $\mathbb{H}^{2} \cap \mathbb{H}_{0}^{1}$. Such subsets are for example the domains with compact regular boundary and convex polygons bounded or unbounded.

As in the $\mathbb{R}^{n}$ case, the following lemma, due to Weinstein, gives us the general behavior of a blowing-up solution of critical mass. 
Lemma 1.2 (Weinstein [21]) Let $u_{k} \in \mathbb{H}^{1}\left(\mathbb{R}^{2}\right)$ be a sequence of functions of critical mass satisfying

$$
\left\{\begin{array}{c}
\beta_{k}=\left\|\nabla u_{k}\right\|_{2} \underset{k \rightarrow \infty}{\longrightarrow} \infty \\
E\left(u_{k}\right) \underset{k \rightarrow \infty}{\longrightarrow} c<\infty
\end{array}\right.
$$

Then there exist points $x_{k} \in \mathbb{R}^{2}$ and $\theta_{k} \in \mathbb{R}$ such that in $\mathbb{H}^{1}\left(\mathbb{R}^{2}\right)$

$$
\frac{e^{i \theta_{k}}}{\beta_{k}} u_{k}\left(\frac{x}{\beta_{k}}+x_{n}\right) \underset{k \rightarrow \infty}{\longrightarrow} \frac{1}{\omega} Q(\omega x)
$$

where $\omega=\|\nabla Q\|_{2}$.

Let $u$ be a solution of $\left(S_{\Omega}\right)$ that blows up at the finite time $T$, that is

$$
\lambda(t)=\frac{\|\nabla u(t)\|_{2}}{\|\nabla Q\|_{2}} \underset{t \rightarrow T}{\longrightarrow} \infty .
$$

By combining Lemma 1.2 for families $u_{k}=u\left(t_{k}\right)$ with $t_{k}$ sequences convergent to $T$ with the result of Kwong on the uniqueness of the ground state ([10]), there exist $\theta(t)$ real numbers and $x(t) \in \mathbb{R}^{2}$ such that in $\mathbb{H}^{1}\left(\mathbb{R}^{2}\right)$

$$
\frac{e^{i \theta(t)}}{\lambda(t)} u\left(t, \frac{x}{\lambda(t)}+x(t)\right) \underset{t \rightarrow T}{\longrightarrow} Q(x)
$$

where $u$ is continuated by zero outside $\Omega$. Then, in the space of distributions,

$$
|u(t, \cdot+x(t))|^{2} \underset{t \rightarrow T}{\longrightarrow}\|Q\|_{2}^{2} \delta_{0}
$$

In this paper we concentrate on the further analysis of the blowing-up solutions with critical mass on a plane domain. The results are the following.

Theorem 1.3 Let $u$ be a $\mathcal{C}\left(\left[0, T\left[, \mathbb{H}_{0}^{1}\right)\right.\right.$ solution of the Schrödinger equation $\left(S_{\Omega}\right)$, which has critical mass and blows up at the finite time $T$.

i) For bounded domains, the blowing-up rate is lower bounded by

$$
\frac{1}{T-t} \lesssim\|\nabla u(t)\|_{2}
$$

ii) If there exist solutions $u$ of critical mass blowing up at a finite time $T$ on the boundary of $\Omega$, that is if the concentration parameter $x(t)$ converges as $t \rightarrow T$ to a point on the boundary, then the blowing-up rate satisfies

$$
\lim _{t \rightarrow T}(T-t)\|\nabla u(t)\|_{2}=\infty
$$

Let us notice that for the Schrödinger equation posed on a domain, the conservations of the mass and of the energy of the solutions are still valid, but the conservation of the derivative of the first momentum and the virial identity fail. In order to avoid these difficulties, we shall use systematically in the proof of Theorem $1.3 \mathrm{a}$ 
Cauchy-Schwarz type inequality derived from Weinstein's inequality. Precisely, we show that if $v$ is a $\mathbb{H}^{1}\left(\mathbb{R}^{2}\right)$ function of critical or subcritical mass, then

$$
\left|\int \Im(v \nabla \bar{v}) \nabla \theta d x\right| \leq\left(2 E(v) \int|v|^{2}|\nabla \theta|^{2} d x\right)^{\frac{1}{2}}
$$

for all real function $\theta$. This inequality allows us to estimate the virial, that we shall assume to be localized if $\Omega$ is unbounded (see Remark 1.6). The lower bound for the blowing-up rate is the same as the one found by Antonini on the torus ([1]).

By following the approach of Weinstein in [22], and the recent results of Maris in [13], we analyze the convergence to the ground state of the modulations of the solutions (1), and we obtain, for bounded domains, the following additional informations.

Proposition 1.4 i) The blow-up rate verifies

$$
\int|u(t)|^{2}|x-x(t)|^{2} d x \approx \frac{1}{\|\nabla u(t)\|_{2}^{2}} .
$$

ii) The concentration parameter $x(t)$ can be chosen to be as the first order momentum

$$
x(t)=\frac{\int|u(t)|^{2} x d x}{\|Q\|_{2}^{2}} .
$$

Corollary 1.5 If the equation $\left(S_{\Omega}\right)$ is considered to be invariant under rotations, then $x(t)$ can be chosen 0 , and we have

$$
g(t) \approx \frac{1}{\|\nabla u(t)\|_{2}^{2}}
$$

Remark 1.6 For unbounded domains, if the solution concentrates at one point, that is if $x(t)$ converges as $t \rightarrow T$, then the first assertion of Theorem 1.3 is true, and so are the assertions of Proposition 1.4, for the virial and the first order momentum localized at the blow-up point.

There is no known example of a solution of nonlinear Schrödinger equation with a blow-up rate larger than $\frac{1}{T-t}$, neither in the case of supercritical mass, nor in the case of supercritical nonlinearities.

Therefore we expect that the blowing-up rate grows exactly like $\frac{1}{T-t}$ and that the profiles are the ones on $\mathbb{R}^{2}$ modulo an exponentially decreasing in $\mathbb{H}^{1}$ function.

Since it is not likely that the blowing-up rate at the boundary grows strictly faster than $\frac{1}{T-t}$, we also expect that there are no solutions blowing-up on the boundary of a domain. This is confirmed for certain simple cases by the following result.

Theorem 1.7 If $\Omega$ is a half-plane or a plane sector, then there are no solutions blowing-up in a finite time on the boundary of the half-plane or in the corner of the sector respectively. 
Indeed, under these geometric hypotheses on $\Omega$, the boundary terms which appear in the second derivative of the virial associated to a blowing-up solution of $\left(S_{\Omega}\right)$ cancel, so we have, as on $\mathbb{R}^{n}$, the virial identity

$$
\partial_{t}^{2} g=16 E(u) .
$$

The proof then follows the one by Merle in [14] for the equation posed on $\mathbb{R}^{n}$, and we obtain that

$$
E\left(e^{i \frac{|x|^{2}}{4(T-t)}} u(t, x)\right)=0,
$$

and by the variational characterization of the ground state, $u(t)$ must be of the type of the critical mass explosive solutions on $\mathbb{R}^{2}$. Therefore we arrive at a contradiction by looking at the support of the solution.

We shall give in the following the proof of the Theorem 1.3. The first section $\$ 2$ contains some results on general domains. We prove the Cauchy-Schwarz type inequality already mentioned, and we calculate the derivative in time for a virial type function. In $\S 3$, by studying the virial, the lower-bound of the blowing-up rate is proved for bounded domains $\Omega$. In $\S 4$, by introducing a localized virial, we find the same lower-bound for the blowing-up rate for unbounded domains. The last section $\$ 5$ contains the results regarding the explosion on the boundary of $\Omega$.

I thank my advisor Patrick Gérard for having introduced me to this beautiful subject and for having guided this work.

\section{Results on general domains}

\subsection{A Cauchy-Schwarz inequality for subcritical mass func- tions}

Lemma 2.1 Let $\theta$ be a real valued function. All $v \in \mathbb{H}^{1}\left(\mathbb{R}^{2}\right)$ with critical or subcritical mass satisfy

$$
(*)\left|\int \Im(v \nabla \bar{v}) \nabla \theta d x\right| \leq\left(2 E(v) \int|v|^{2}|\nabla \theta|^{2} d x\right)^{\frac{1}{2}}
$$

Proof. The precised version of the Gagliardo-Nirenberg inequality, presented in the introduction, is, for function $w$ in $\mathbb{H}^{1}\left(\mathbb{R}^{2}\right)$,

$$
\|w\|_{4}^{4} \leq \frac{2}{\|Q\|_{2}^{2}}\|w\|_{2}^{2}\|\nabla w\|_{2}^{2}
$$

As a consequence, if

$$
\|w\|_{2} \leq\|Q\|_{2}
$$

then the energy of $w$ is positive.

Therefore on one hand

$$
0 \leq E\left(e^{i \alpha \theta} v\right)
$$

for every real number $\alpha$ and for all real function $\theta$. On the other hand

$$
E\left(e^{i \alpha \theta} v\right)=\frac{1}{2} \int|i \alpha \nabla \theta v+\nabla v|^{2} d x-\frac{1}{4} \int|v|^{4} d x=
$$




$$
=\frac{\alpha^{2}}{2} \int|v|^{2}|\nabla \theta|^{2} d x-\alpha \int \Im(v \nabla \bar{v}) \nabla \theta d x+E(v)
$$

Thus the discriminant of the equation in $\alpha$ must be non positive and we obtain the claimed Cauchy-Schwarz type inequality $(*)$.

\subsection{Derivatives of virial type functions}

Let $u$ be a solution of $\left(S_{\Omega}\right)$ and let $h$ be a $\mathcal{C}^{\infty}\left(\mathbb{R}^{2}\right)$ function. Then, by using the fact that $u$ satisfies $\left(S_{\Omega}\right)$, we obtain

$$
\partial_{t} \int_{\Omega}|u(t)|^{2} h d x=2 \int_{\Omega} \Re\left(u(t) \bar{u}_{t}(t)\right) h d x=2 \int_{\Omega} \Im\left(u(t) \Delta \bar{u}_{t}(t)\right) h d x .
$$

Since $u$ cancels on the boundary of $\Omega$, by integration by parts

$$
\partial_{t} \int_{\Omega}|u(t)|^{2} h d x=-2 \int_{\Omega} \Im\left(u(t) \nabla \bar{u}_{t}(t)\right) \nabla h d x .
$$

\section{The blow-up rate on bounded plane domains}

\subsection{The convergence of the concentration points $x(t)$}

From (2) it follows that for a test function $\psi$,

$$
\int_{\Omega-x(t)}|u(t, x+x(t))|^{2} \psi(x) d x \underset{t \rightarrow T}{\longrightarrow}\|Q\|_{2}^{2} \psi(0) .
$$

If $\psi$ is chosen such that $\psi(0) \neq 0$ then, since the set $\Omega$ is bounded, it follows that

$$
\limsup _{t \rightarrow T}|x(t)|<\infty .
$$

We shall show that $x(t)$ has a limit at the time $T$.

The first order momentum

$$
f(t)=\int_{\Omega}|u(t, x)|^{2} x d x,
$$

stays finite in time since $\Omega$ is bounded and $u$ conserves its mass. By using the formula (3) for vector-valued functions $h$, one can calculate the derivative

$$
f^{\prime}(t)=-2 \int_{\Omega} \Im(u(t) \nabla \bar{u}(t)) d x .
$$

The inequality $(*)$ in the special case $\theta_{i}(x)=x_{i}$ implies that this derivative is bounded in time

$$
\left|f^{\prime}(t)\right|^{2} \leq 4 \sum_{i \in\{1,2\}}\left|\int_{\Omega} \Im(u(t) \nabla \bar{u}(t)) \nabla \theta_{i} d x\right|^{2} \leq 16 E(u)\|u\|_{2}^{2} .
$$


Therefore $f$ admits a limit at the time $T$. Let us define $x_{0}$ by

$$
f(T)=x_{0}\|Q\|_{2}^{2} .
$$

Using the convergence (2) and the fact that $\Omega-x(t)$ is a uniformly bounded set, one has

$$
f(t)-x(t)\|Q\|_{2}^{2}=\int_{\Omega-x(t)}|u(t, x+x(t))|^{2} x d x \underset{t \rightarrow T}{\longrightarrow} 0 .
$$

Therefore the point $x_{0}$ is the limit of $x(t)$, and the square of the solution behaves like a Dirac function

$$
|u(t, \cdot)|^{2} \underset{t \rightarrow T}{\longrightarrow}\|Q\|_{2}^{2} \delta_{x_{0}} .
$$

Up to a translation, one can suppose that the solution blows up in $0 \in \bar{\Omega}$.

\subsection{Lower bound for the blow-up rate}

The derivative in time of the the virial,

$$
g(t)=\int_{\Omega}|u(t, x)|^{2}|x|^{2} d x
$$

can be calculated with the formula (3) with $h(x)=|x|^{2}$, and

$$
g^{\prime}(t)=-4 \int_{\Omega} \Im(u(t) \nabla \bar{u}(t)) x d x .
$$

Therefore the inequality $(*)$ in the case $\theta(x)=|x|^{2}$ implies that

$$
\left|g^{\prime}(t)\right| \leq 4 \sqrt{2 E(u) g(t)} .
$$

The concentration result (4) of the former subsection gives

$$
g(T)=0
$$

and one can now write

$$
\sqrt{g(t)}=-\int_{t}^{T} \frac{g^{\prime}(\tau)}{2 \sqrt{g(\tau)}} d \tau \leq \int_{t}^{T} 2 \sqrt{2 E(u)}=2 \sqrt{2 E(u)}(T-t)
$$

and obtain

$$
g(t) \leq 8 E(u)(T-t)^{2} .
$$

Then the uncertainty principle

$$
\left(\int_{\mathbb{R}^{2}}|u|^{2}\right)^{2} \leq\left(\int_{\mathbb{R}^{2}}|u|^{2}|x|^{2}\right)\left(\int_{\mathbb{R}^{2}}|\nabla u|^{2}\right)
$$

gives us a lower bound of the blow-up rate

$$
\frac{\|Q\|_{2}^{2}}{2 \sqrt{2 E(u)}(T-t)} \leq\|\nabla u(t)\|_{2}
$$

so the first assertion of Theorem 4.1.2 is proved. 


\section{The blow-up rate on unbounded plane domains}

Consider now the equation $\left(S_{\Omega}\right)$ on an unbounded domain of the plane or on a surface. Let $u$ be a critical mass solution that blows up in an interior point $x_{0}$ of $\Omega$, that is

$$
x(t) \underset{t \rightarrow T}{\longrightarrow} x_{0} .
$$

Up to a translation, we can suppose that $x_{0}$ is zero and so,

$$
|u(t, x)|^{2} \underset{t \rightarrow T}{\longrightarrow}\|Q\|_{2}^{2} \delta_{0} .
$$

Let $\phi$ be a $\mathcal{C}_{0}^{\infty}$ function, equal to 1 on $B(0, R)$. Let us introduce the localized virial of the solution

$$
g_{\phi}(t)=\int|u(t, x)|^{2} \phi^{2}(x)|x|^{2} d x .
$$

Then, using (3) with $h(x)=\phi^{2}(x)|x|^{2}$, one has

$$
g_{\phi}^{\prime}(t)=-2 \int \Im(u(t) \nabla \bar{u}(t)) \nabla\left(\phi^{2}|x|^{2}\right) d x .
$$

The inequality $(*)$ with $\theta(x)=\phi^{2}(x)|x|^{2}$ gives us

$$
\left|g_{\phi}^{\prime}(t)\right|^{2} \leq 8 E(u) \int|u|^{2}\left|\nabla\left(\phi^{2}|x|^{2}\right)\right|^{2} d x
$$

Since $\nabla\left(\phi^{2}|x|^{2}\right)$ is a $\mathcal{C}_{o}^{\infty}\left(\mathbb{R}^{2}\right)$ function cancelling at 0 , and since the square of $|u|$ behaves like a Dirac distribution, it follows that

$$
g_{\phi}^{\prime}(T)=0
$$

Then, as in the former section, and using the existence of a positive constant $C$ such that

$$
\left|\nabla\left(\phi^{2}|x|^{2}\right)\right|^{2} \leq C \psi^{2}|x|^{2}
$$

one has

$$
g_{\phi}(t) \lesssim(T-t)^{2}
$$

The uncertainty principle reads

$$
\left(\int|u|^{2} \phi^{2} d x\right)^{2} \leq\left(\int|u|^{2} \phi^{2}|x|^{2} d x\right)\left(\int|\nabla(u \phi)|^{2} d x\right) .
$$

By integrating by parts the last term and by using the fact that $\phi$ is equal to 1 on $B(0, R)$, it follows that

$$
\left(\int_{B(0, R)}|u(t)|^{2}\right)^{2} \leq g_{\phi}(t)\left(\int|\nabla u|^{2} \phi^{2} d x-\int|u|^{2} \phi \Delta \phi d x\right) .
$$

Since $\phi$ is a $\mathcal{C}_{0}^{\infty}$ function,

$$
\left(\int_{B(0, R)}|u(t)|^{2}\right)^{2} \leq g_{\phi}(t)\left(C \int|\nabla u|^{2} d x-\int|u|^{2} \phi \Delta \phi d x\right) .
$$


On the one hand the $\mathbb{L}^{2}$ norm of $u$ is conserved. On the other hand, the behavior of $|u|^{2}$ as a Dirac distribution implies that the norm of its restriction outside a neighborhood of zero tends to 0 in time. So we have

$$
\left\{\begin{array}{c}
\int_{B(0, R)}|u(t)|^{2}=O(1) \\
\int|u(t)|^{2} \phi \Delta \phi d x=o(1)
\end{array},\right.
$$

and since $g_{\phi}$ is bounded in time,

$$
1 \lesssim \sqrt{g_{\phi}(t)}\|\nabla u(t)\|_{2}
$$

Then the decay of $g_{\phi}$ gives us the lower bound of the blow-up speed

$$
\frac{1}{T-t} \lesssim\|\nabla u(t)\|_{2}
$$

and the first assertion of Theorem 4.1.2 is completely proved.

\section{Blow-up on the boundary}

\subsection{Necessary condition for blow-up on the boundary}

Let us first introduce a notion of limit of sets, as in [5].

Definition $1 A$ sequence of open sets $M_{m}$ is said to tend to an open set $M$ of $\mathbb{R}^{2}$ if the following conditions are verified.

i) For all compact $K \subset M$, there exist $n_{K} \in \mathbb{N}$, such that for all $n \geq n_{K}, K \subset M_{n}$.

ii) For all compact $K \subset^{c} \bar{M}$, there exist $n_{K} \in \mathbb{N}$, such that for all $n \geq n_{K}, K \subset^{c} \overline{M_{n}}$.

Let us suppose that there exists an explosive solution $u$ of the equation $\left(S_{\Omega}\right)$ at $0 \in \partial \Omega$. The convergence (1) implies that

$$
\lambda(t)(\Omega-x(t)) \underset{t \rightarrow T}{\longrightarrow} \mathbb{R}^{2} .
$$

As in [5], the limit set depends on the position of $x(t)$ with respect to the boundary of $\Omega$. If there is a positive number $C$ such that for all $t$

$$
\lambda(t) d(x(t), \partial \Omega) \leq C,
$$

then $\lambda(t)(\Omega-x(t))$ tends to a half-plane and blow-up cannot occur. Also, if

$$
\lambda(t) d(x(t), \partial \Omega) \underset{t \rightarrow T}{\longrightarrow} \infty,
$$

and $x(t)$ is not in $\Omega$, then $\lambda(t)(\Omega-x(t))$ is a set that moves to infinity and does not cover at the limit time the whole plane. Therefore the only possibility to have explosion on the boundary is that $x(t) \in \Omega$ and

$$
\lambda(t) d(x(t), \partial \Omega) \underset{t \rightarrow T}{\longrightarrow} \infty .
$$


In particular, since 0 is on the boundary,

$$
|\lambda(t) x(t)| \underset{t \rightarrow T}{\longrightarrow} \infty
$$

We have

$$
|x(t)|^{2} \int_{B\left(x(t), \frac{C}{\lambda(t)}\right)}|u|^{2} \leq 2 \int_{B\left(x(t), \frac{C}{\lambda(t)}\right)}|u|^{2}|x-x(t)|^{2}+2 \int_{B\left(x(t), \frac{C}{\lambda(t)}\right)}|u|^{2}|x|^{2} .
$$

On the one hand, by using the Weinstein relation (1), one has

$$
|x(t)|^{2} \int_{B\left(x(t), \frac{C}{\lambda(t)}\right)}|u|^{2} \approx|x(t)|^{2}
$$

On the other hand, using again (1),

$$
\int_{B\left(x(t), \frac{C}{\lambda(t)}\right)}|u|^{2}|x-x(t)|^{2} \lesssim \frac{1}{\lambda(t)^{2}}
$$

In view of (5), these two facts imply

$$
|x(t)|^{2} \lesssim \int_{B\left(x(t), \frac{C}{\lambda(t)}\right)}|u|^{2}|x|^{2} \lesssim g_{\psi}
$$

where $g_{\psi}$ is the localized virial function defined in $§ 4.4 .2$. In the previous section it was proved that

$$
g_{\psi} \lesssim(T-t)^{2}
$$

so it follows that

$$
|x(t)| \lesssim T-t
$$

By using again (5),

$$
\frac{1}{T-t} \ll \lambda(t),
$$

and the second assertion of Theorem 4.1.1 is proved.

\section{References}

[1] C. Antonini, Lower bounds for the $\mathbb{L}^{2}$ minimal periodic blow-up solutions of critical nonlinear Schrödinger equation, Diff. Integral Eq. 15 (2002), no. $6,749-768$.

[2] H. Brézis, T. Gallouët, Nonlinear Schrödinger evolution equation, Nonlinear Analysis, Theory Methods Appl. 4 (1980), no. 4, 677-681.

[3] N. Burq, P. Gérard, N. Tzvetkov, Two singular dynamics of the nonlinear Schrödinger equation on a plane domain, Geom. Funct. Anal. 13 (2003), $1-19$. 
[4] T. Cazenave, An introduction to nonlinear Schrödinger equations, Textos de Métodos Matemáticos 26, Instituto de Matemática-UFRJ, Rio de Janeiro, RJ (1996).

[5] I. Gallagher, P. Gérard, Profile decomposition for the wave equation outside a convex obstacle, J. Math. Pures Appl. (9) 80 (2001), no. 1, 1-49.

[6] J. Ginibre, G. Velo, On a class of Schrödinger equations. I. The Cauchy problem, general case, J. Funct. Anal. 32 (1979), no. 1, 1-71.

[7] R. T. Glassey, On the blowing up of solutions to the Cauchy problem for nonlinear Schrödinger equations, J. Math. Phys. 18 (1977), no. 9, 1794-1797.

[8] T. Kato, On nonlinear Schrödinger equations, Ann. I. H. P. Physique Théorique 46 (1987), no. 1, 113-129.

[9] O. Kavian, A remark on the blowing-up of solutions to the Cauchy problem for nonlinear Schrödinger equations, Trans. Amer. Math. Soc. 299 (1987), no. $1,193-203$.

[10] M. K. Kwong, Uniqueness of positive solutions of $\Delta u-u+u^{p}=0$ in $\mathbb{R}^{N}$, Arch. Rat. Mech. Ann. 105 (1989), no. 3, 243-266.

[11] P. L. Lions, The concentration-compactness principle in the calculus of variations. The locally compact case. I, Ann. Inst. H. Poincaré Anal. Non Linéaire 1 (1984), no. 2, 109-145.

[12] P. L. Lions, The concentration-compactness principle in the calculus of variations. The locally compact case. II, Ann. Inst. H. Poincaré Anal. Non Linéaire 1 (1984), no. 4, 223-283.

[13] M. Maris, Existence of nonstationary bubbles in higher dimensions, J. Math. Pures. Appl. 81 (2002), 1207-1239.

[14] F. Merle, Determination of blow-up solutions with minimal mass for nonlinear Schrödinger equation with critical power, Duke Math. J. 69 (1993), no. $2,427-454$.

[15] F. Merle, P. Raphaël, Blow-up dynamic and upper bound on blow-up rate for critical non linear Schrödinger equation, Université de Cergy-Pontoise, preprint (2003).

[16] F. Merle, P. Raphaël, On blow-up profile for critical non linear Schrödinger equation, Université de Cergy-Pontoise, preprint (2003).

[17] T. Ogawa, T. Ozawa, Trudinger type inequalities and uniqueness of weak solutions for the nonlinear Schrödinger equations, J. Math. Anal. Appl. 155 (1991), no. 2, 531-540. 
[18] T. Ogawa, Y. Tsutsumi, Blow-up solutions for the nonlinear Schrödinger equation with quartic potential and periodic boundary conditions, Springer Lecture Notes in Math. 1450 (1990), 236-251.

[19] M. V. Vladimirov, On the solvability of mixed problem for a nonlinear equation of Schrödinger type, Dokl. Akad. Nauk SSSR 275 (1984), no. 4, 780-783.

[20] M. I. Weinstein, Nonlinear Schrödinger equations and sharp interpolate estimates, Comm. Math. Phys. 87 (1983), no. 4, 567-576.

[21] M. I. Weinstein, On the structure and formation of singularities in solutions to nonlinear dispersive evolution equations, Comm. Part. Diff. Eq. 11 (1986), no. $5,545-565$.

[22] M. I. Weinstein, Modulation stability of ground states of nonlinear Schrödinger equations, Siam. J. Math. Anal. 16 (1985), no. 3, 472-491.

[23] V. E. Zakharov, Collapse of Lagmuir waves, Sov. Phys. JETP 35 (1972), 908-914.

Université de Paris Sud, Mathématiques, BÂt.425, 91405 Orsay

Valeria.Banica@math.u-psud.fr 